ginal Paper

\title{
Protein Kinase C Activation Inhibits Rat and Human Hyperpolarization Activated Cyclic Nucleotide Gated Channel (HCN)1 - Mediated Current in Mammalian Cells
}

\author{
Olivia Reetz Ulf Strauss
}

Institute of Cell Biology and Neurobiology, Center for Anatomy, Charité-Universitätsmedizin Berlin, Berlin

\section{Key Words}

Protein kinase C • Hyperpolarization-activated cyclic nucleotide-gated channel • HEK293 cells - N1E-115 cells

\begin{abstract}
Background/Aims: Hyperpolarization activated cyclic nucleotide gated 1 (HCN1) channels determine neuronal excitability in several brain regions. In contrast to HCN2 and HCN4, HCN1 is less sensitive to CAMP and the number of other known modulators is limited. One of those, the protein kinase C (PKC), showed opposing effects on mouse HCN1 channels expressed in Xenopus oocytes. Methods: In order to study PKC effects on HCN1 mediated currents in a mammalian environment we expressed rat HCN1 or human HCN1 in human embryonic kidney (HEK293) cells and rat HCN1 in murine neuroblastoma (N1E-115) cells. We recorded the resulting $I_{\mathrm{h}}$ before and during the application of the membrane permeable nonmetabolizable PKC-activator 4ßPMA in cell-attached mode of the patch-clamp technique, leaving the intracellular environment intact. Results: $4 \beta P M A$ reduced maximal HCN1 mediated currents to about $60-70 \%$ and slowed its activation, but left its voltage sensitivity unchanged. The effect was neither due to species-related differences nor restricted to HEK293 cells, because it was comparable for human and rat HCN1 in HEK293 and for rat HCN1 in N1E-115 cells. However, pre-treatment with the PKC blocker GF109203X abolished 4BPMA induced $I_{\mathrm{h}}$ changes. Disrupting the intracellular environment by recording in whole-cell mode drastically reduced the 4ßPMA effect. Conclusion: PKC activation reduces and slows $I_{\mathrm{h}}$ in non-neuronal and neuronal mammalian cells transfected with rat or human HCN1 if the intracellular content remains intact.
\end{abstract}

Copyright $\odot 2013$ S. Karger AG, Basel 


\section{Introduction}

Hyperpolarization-activated, cyclic nucleotide-gated (HCN) channels underlying $I_{\mathrm{h}}$ regulate the neuronal excitability in many regions of the mammalian brain including the neocortex and hippocampus [1]. In these regions, HCN1, as one of the four tetramer-forming subunits found in mammals, is predominant in pyramidal neurons [2]. In general, known determinants of HCN1 function - and therewith neocortical excitability - include total protein expression, surface expression, channel properties and tetramer formation [1]. Fast alterations in HCN1 mediated currents, in particular, likely arise from changes in channel properties such as gating. Although the gating of HCN1 channels is virtually insensitive to the canonical HCN channel modulator cAMP, a depolarizing shift in HCN1 voltage dependence can occur apart from the action of cyclic nucleotides in Xenopus oocytes by e. g. allosteric gating by phospatidylinositol-4,5-biphosphate [3] or by protein kinase C (PKC) activation

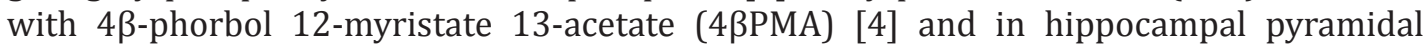
neurons by p38MAP kinase [5]. However, changes in the maximum current without shifts in voltage dependence can likewise underlie fast alterations of HCN1 mediated currents [6, 7]. Interestingly, the activation of conventional and novel isoforms of PKC by $4 \beta P M A$ affected both, gating and maximum current, of HCN1 channels expressed in Xenopus oocytes, although in an opposing manner [4]. In the Xenopus expression system PKC activation depolarized voltage sensitivity of HCN1 mediated currents, but suppressed its maximum. Therewith, the net effect - apart from the newly opened voltage window - remains unclear. In order to expand the knowledge on this HCN1 modulation and, in particular, to test the PKC action in a mammalian system, we chose the well characterized rat (r)HCN1 [6] and human (h)HCN1 [8] expressed in HEK293 cells. A synopsis of our data suggests that PKC overall inhibits HCN1mediated currents in mammalian cells due to a reduction in maximal current. In contrast to data on murine HCN1 expressed in Xenopus oocytes PKC does not influence gating.

\section{Materials and Methods}

\section{Cell culture and transfection}

HEK293 cells (DSMZ, Braunschweig, Germany) or N1E-115 cells (ATCC, Wesel, Germany) were cultured at $37^{\circ} \mathrm{C}\left(95 \% \mathrm{O}_{2}\right.$ and $\left.5 \% \mathrm{CO}_{2}\right)$ in DMEM (Dulbecco's modified eagle medium) including $4.5 \mathrm{~g} / \mathrm{L} \mathrm{D}$-Glucose, L-glutamine and $3.7 \mathrm{~g} / \mathrm{L} \mathrm{NaHCO}_{3}$ supplemented with $10 \%$ fetal calf serum, $1 \%$ penicillin/streptomycin (all from Pan biotech GmbH, Aidenbach, Germany) and $1 \%$ L-glutamine (for N1E-115 cells DMEM with pyruvate instead of $\mathrm{NaHCO}_{3}$ and without supplemental L-glutamine). Cells were detached by use of $2.5 \%$ trypsin (life technologies GmbH, Darmstadt, Germany) and passaged regularly. One day after HEK293 cells or N1E-115 cells were plated at low density on poly-L-lysine coated cover slips they were transfected with pIRES2_dsRed-rHCN1 (NM_053375.1) or with (human) hHCN1 (in pcDNA 3.1; kind gift of Juliane Stieber) in a co-transfection with pIRES2_eGFP (3:1 ratio) by $2 \mathrm{M} \mathrm{CaPO}_{4}$ for $15 \mathrm{~min}$ as described previously [6]. In the used rHCN1 construct the base pairs 75 - 2807 exactly resembled the respective published sequence of rHCN 1 as proofed by sequencing. Culture passages from 1 to 20 were used.

\section{Patch-Clamp Recordings}

2 - 4 days after transfection HEK293 cells (for N1E-115 cells 2 - 3 days after transfection) on their cover slips were placed into a recording chamber. The actual $I_{\mathrm{h}}$ magnitude (the channel presence in the membrane) appeared tightly related to the time after transfection and the fluorescent dye (see below) seemed to negatively influence cell viability, leaving only a small time frame for measurements. Cells were constantly superfused with extracellular solution comprising (in $\mathrm{mM}$ ): $120 \mathrm{NaCl}, 10 \mathrm{KCl}, 0.5 \mathrm{MgCl}_{2}, 10$ HEPES, 10 glucose (all from Carl Roth GmbH and Co. KG, Karlsruhe, Germany), $1.8 \mathrm{CaCl}_{2}$ (Merck, Darmstadt, Germany) 10 TEA and 2.5 4-aminopyridine (both from Sigma-Aldrich, Munich, Germany) (pH adjusted with $\mathrm{NaOH}$ to 7.4). HEK293 cells expressing rHCN1 were identified by red fluorescence with an inverted microscope (Axiovert S100; Zeiss, Oberkochen, Germany). Pipettes were pulled (P-97 micropipette puller; Sutter Instruments, Novato, CA, USA) to a resistance of 2.0 - 3.5 M $\Omega$. The intracellular solution comprised (in 
mM) 120 K-gluconate, 10 Na-phosphocreatine, 11 EGTA, 2 Mg$^{2+}$ ATP, 0.3 Tris-GTP (Sigma-Aldrich), $10 \mathrm{KCl}, 1$ $\mathrm{MgCl}_{2}, 1 \mathrm{CaCl}_{2}, 10$ HEPES, adjusted with KOH (Carl Roth) to $\mathrm{pH} 7.2$ and was kept on ice for one day. For some whole cell experiments on HEK293 cells a modified cAMP containing intracellular solution was used (in mM): 120 K-methylsulphate $\left(\mathrm{KMeSO}_{4}\right.$ ) (ICN Biomedical Inc, California, USA), $20 \mathrm{KCl}, 14 \mathrm{Na}$-phosphocreatine, $4 \mathrm{NaCl}, 0.5$ EGTA, 10 HEPES, $4 \mathrm{Mg}^{2+}$-ATP, 0.3 Tris-GTP and 0.1 cAMP (Sigma-Aldrich) (pH adjusted with $\mathrm{KOH}$ to 7.2). Experiments were conducted at room temperature and recorded with an EPC-10 amplifier (HEKA, Lambrecht, Germany) controlled by PatchMaster v2.32 software (HEKA). 4 $\beta$-phorbol 12-myristate 13-acetate (4ßPMA) (R\&D Systems GmbH, Wiesbaden-Nordenstadt, Germany) was dissolved in $881.68 \mu \mathrm{l}$ of 99.8 \% DMSO (Sigma-Aldrich) and GF109203X (R\&D Systems) was dissolved in $2.35 \mathrm{ml} 99.8 \%$ DMSO to a stock concentration of $10 \mathrm{mM}$. Stocks were diluted with extracellular solution to a final concentration of 0.2 $\mu \mathrm{M}$ or $1 \mu \mathrm{M} 4 \beta \mathrm{PPMA}$ or GF109203X / $0.02 \%$ or $0.1 \%$ DMSO respectively.

\section{Data and Statistical Analysis}

Data were analyzed with FitMaster (HEKA) and Origin 8.5 (Origin Labs, Northampton, MA, USA). For the analysis of the experiments in whole cell mode, recordings with a holding current larger than $200 \mathrm{pA}$ were excluded. An offline linear leak subtraction was performed only for estimating the $I_{\mathrm{h}}$ amplitude in cells measured in whole cell mode. In cell attached mode the leak current was negligible, so no leak subtraction was applied. For analysis of the voltage dependence of the current, tail current amplitudes of all voltage steps were normalized separately for each cell and plotted against the preceding voltage step. Each data set was fitted with a Boltzmann function: $I(V)=\left(\left[A_{1}-A_{2}\right] / 1+e\left(\left[V-V_{1 / 2}\right] / k\right)+A_{2}\right)$ resulting in the floating parameters $\mathrm{V}_{1 / 2}$, the voltage at which half of the maximal current was activated and $\mathrm{k}$, the slope of the fitted curve. Calculated liquid junction potentials of $9.3 \mathrm{mV}$ were not corrected for. The activation kinetics were fitted in cell attached recordings by a single exponential equation $y(x)=y_{0}+A_{1}\left(1-e^{-x / \tau 1}\right)$ and for the whole cell recordings by a double exponential equation $y(x)=y_{0}+A_{1}\left(1-e^{-x / \tau 1}\right)+A_{2}\left(1-e^{-x / \tau 2}\right)$ (as integrated in FitMaster), resulting in one or two (fast and slow) time constants $\tau$. Statistical tests were performed with the nonparametric test for paired data (Wilcoxon signed rank test) in case of non-normal distribution and with the paired $\mathrm{t}$-test in case of normal distribution. Results were regarded as statistical significant if $\mathrm{P}<$ 0.05. Error bars represent SEM.

\section{Results}

4ßPMA reduced and slowed rHCN1 or hHCN1 mediated $I_{h}$ in HEK293 cells

To study PKC - HCN1 interactions in a mammalian system we utilized human embryonic kidney (HEK) 293 cells transfected with $\mathrm{rHCN} 1$ or hHCN1. By recording $I_{\mathrm{h}}$ in the cell-attached mode we aimed to conserve the intracellular content and the respective signaling pathways in particular. Under those conditions $I_{\mathrm{h}}$ conductance for rHCN1 ranged between 60 and 880 pS. Given a single rHCN1 channel conductance of 680 fS [9] we simultaneously recorded from 88 to 1294 rHCN1 channels. Bath applying the membrane permeable 4ßPMA enabled us to activate the intracellular PKC without affecting the extracellular HCN1 domains under the attached pipette. Such treatment reduced the maximal rHCN1 mediated current to about $73 \%$ : the application of $1 \mu \mathrm{M}$ reduced the maximal $I_{\mathrm{h}}$ amplitude from $I_{\text {hctrl }}=51.7 \pm 11.0 \mathrm{pA}$ to $I_{\text {h } 4 \beta \mathrm{PMA}}=37.9 \pm 8.0 \mathrm{pA}(\mathrm{n}=6$, Wilcoxon signed-rank test: $P<0.05 ;$ Fig. $1 \mathrm{~A} / \mathrm{B})$ and $0.2 \mu \mathrm{M}$ from $I_{\text {hctrl }}=45.8 \pm 6.6 \mathrm{pA}$ to $I_{\mathrm{h} 4 \beta \mathrm{PMA}}=33.6 \pm 7.5 \mathrm{pA}(\mathrm{n}=8$, paired t- test, $P=0.003)$. Although the currents were relatively small, their individual amplitude was not correlated to the magnitude of the 4ßPMA effect $(r=-0.22)$.

The $I_{\mathrm{h}}$ reduction was accompanied by a slowing of $I_{\mathrm{h}}$ activation (Fig. 1B). The rHCN1 current activation was best fitted with a single exponential equation at $-130 \mathrm{mV}$. The resulting time constant increased from $\tau_{\text {ctrl }}=66 \pm 16.0 \mathrm{~ms}$ under control conditions to $\tau_{\text {ABPMA }}$ $=104.7 \pm 25.9 \mathrm{~ms}$ during $1 \mu \mathrm{M} 4 \beta \mathrm{PMA}$ application $(P<0.05$; Fig. $1 \mathrm{C} / \mathrm{D})$ and from $\tau_{\mathrm{ctrl}}=85 \pm$ $9.9 \mathrm{~ms}$ under control conditions to $\tau_{4 \beta \mathrm{PMA}}=95.4 \pm 12.2 \mathrm{~ms}$ during $0.2 \mu \mathrm{M} 4 \beta \mathrm{PMA}$ application $(P<0.05)$. However, in contrast to the two-electrode voltage clamp recordings in Xenopus oocytes [4], 4 $\beta$ PMA did not change the voltage of half maximal current activation $\left(\mathrm{V}_{1 / 2}\right)$ by $\left(\mathrm{V}_{1 / 2 \mathrm{ctrl}}=-96.0 \pm 3.3 \mathrm{mV}\right.$ vs. $\mathrm{V}_{1 / 24 \beta \mathrm{PMA}}=-99.0 \pm 3.0 \mathrm{mV}, \mathrm{n}=6$, Wilcoxon signed-rank test: 
A

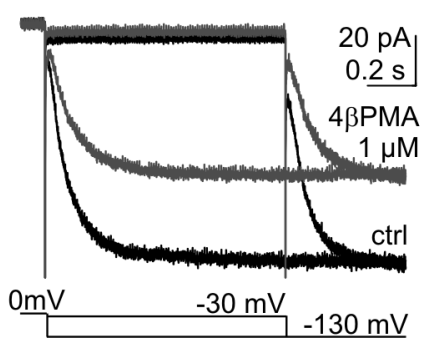

C

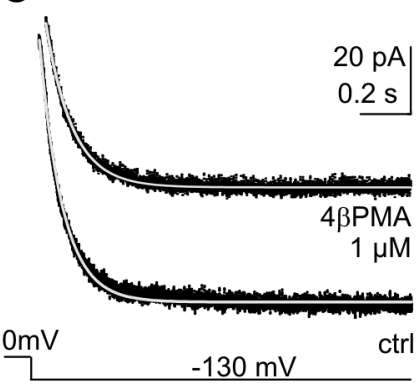

E

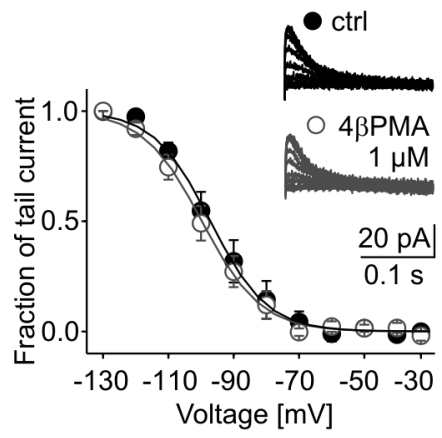

B

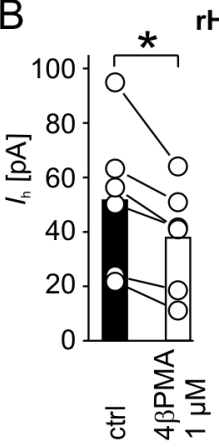

rHCN1

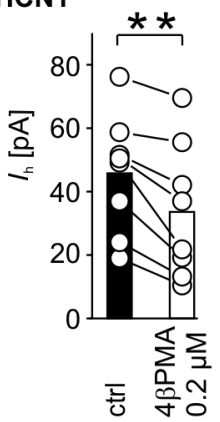

D
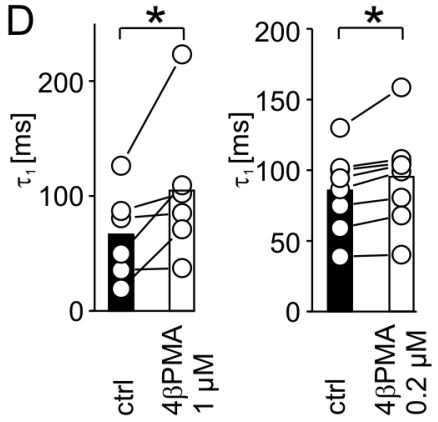

$\mathrm{F}$

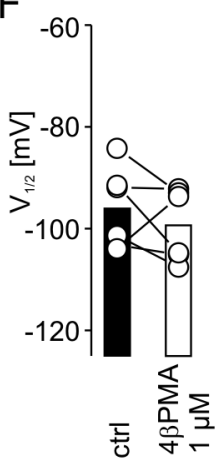

(C) 2013 S. Karger AG, Basel

www.karger.com/cpb
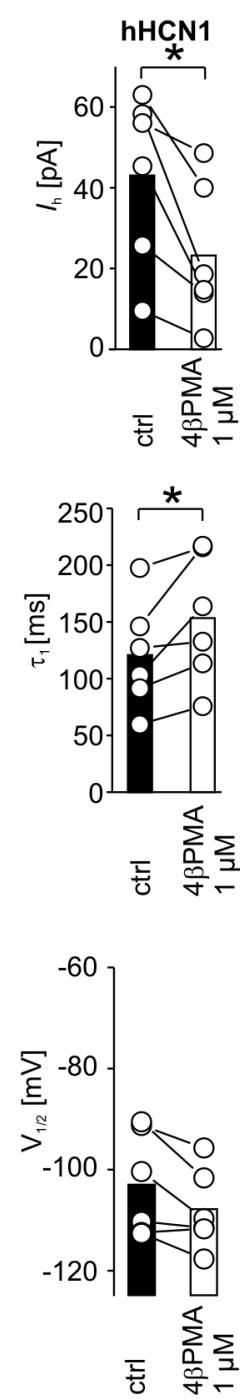

Fig. 1. 4 3 PMA induced PKC activation reduced maximum rHCN1 or hHCN1 mediated $I_{\mathrm{h}}$ and slowed their activation in mammalian cells with intact intracellular content. A. Traces of rHCN1 mediated $I_{\mathrm{h}}$ at voltage steps of $-30 \mathrm{mV}$ and $-130 \mathrm{mV}$ as indicated in the pulse protocol at the bottom recorded in HEK293 cells in cell attached mode before (black trace) and during the application of $1 \mu \mathrm{M} 4 \beta \mathrm{PMA}$ (grey trace). B. Population data of maximum $I_{\mathrm{h}}$ measured at the end of a 1-sec pulse to $-130 \mathrm{mV}$ demonstrate the decrease of $I_{\mathrm{h}}$ after $4 \beta P M A$ addition. C. Distinct data points represent the time course of $I_{\mathrm{h}}$ activation at $-130 \mathrm{mV}$ under control conditions (lower trace) and after 4 $\beta$ PMA application (upper trace). Both are mathematically described by a single exponential function (light grey line). D. Population data of time constants of $I_{\mathrm{h}}$ activation reveal a considerable slowing. E. Voltage dependence of $I_{\mathrm{h}}$ activation. The tail currents after different preceding command potentials (insets) were normalized to the maximal tail current for each cell. For the graph values were averaged, plotted against the preceding voltage and fitted with the Boltzmann equation. F. Population data of half maximal current activation voltages $\left(\mathrm{V}_{1 / 2}\right)$ estimated for individual cells before and during $4 \beta$ PMA application. Control values are represented as filled black, values under 4 $\beta$ PMA as open white columns throughout all figures. *denotes significance level $\mathrm{P}<0.05$ throughout all figures.

$P=0.3 ;$ Fig. $1 \mathrm{E} / \mathrm{F}$ under $1 \mu \mathrm{M} 4 \beta \mathrm{PMA}$ or $\mathrm{V}_{1 / 2 \mathrm{ctrl}}=-98.0 \pm 3.0 \mathrm{mV}$ vs. $\mathrm{V}_{1 / 24 \beta \mathrm{PMA}}=-101.9 \pm 1.7$ $\mathrm{mV} \mathrm{n}=8$, paired t-test: $P=0.07$ under $0.2 \mu \mathrm{M} 4 \beta \mathrm{PMA}$ ). These data suggest that activation of PKC results in a suppression of maximal rHCN1 mediated current accompanied by a slowing of its activation. 
Fig. 2. $4 \beta P M A$ affects $r H C N 1$ in N1E115 cells as in HEK293 cells. A. Traces of rHCN1 mediated $I_{\mathrm{h}}$ in N1E-115 cells at voltage steps of $-30 \mathrm{mV}$ and $-130 \mathrm{mV}$ recorded under the same conditions as in HEK293 cells before (black trace) and after the application of $1 \mu \mathrm{M} 4 \beta \mathrm{PMA}$ (grey trace). B. Population data of maximum $I_{\mathrm{h}}$ amplitude measured at $-130 \mathrm{mV}$ demonstrate the decrease of $I_{\mathrm{h}}$ by $1 \mu \mathrm{M}$ $4 \beta P M A$ (left); of single exponential function time constants of $I_{\mathrm{h}}$ activation reveal a considerable slowing by $1 \mu \mathrm{M}$ $4 \beta$ PMA (middle); and of unperturbed $\mathrm{V}_{1 / 2} \mathrm{~S}$ estimated for individual cells under control conditions and under $1 \mu \mathrm{M} 4 \beta \mathrm{PMA}$ (right).

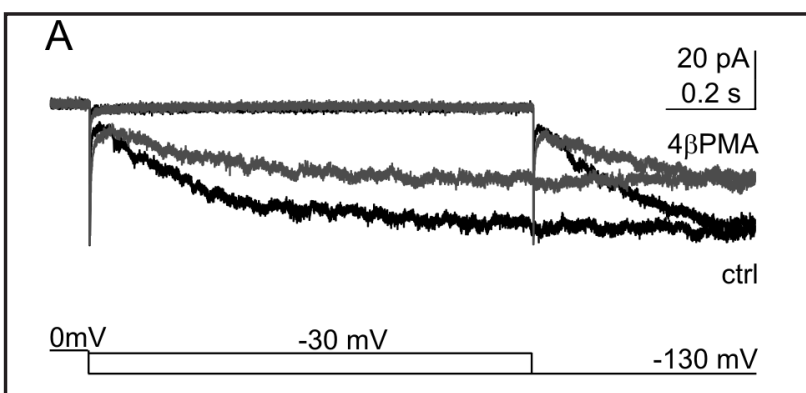

B
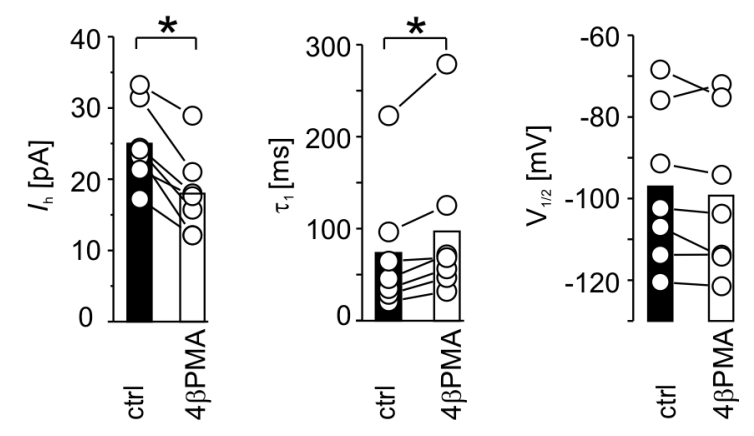

In order to investigate whether species related differences or incompatibilities influenced our results, we subsequently transfected HEK293 cells with hHCN1. Analysis of the resulting currents yielded a comparable effect of 4ßPMA on rHCN1 and hHCN1 mediated $I_{\mathrm{h}}: 1 \mu \mathrm{M} 4 \beta \mathrm{PMA}$ reduced the maximal hHCN1 mediated $I_{\mathrm{h}}$ amplitude to about $57 \%$ from $I_{\text {hctrl }}=43 \pm 8.7 \mathrm{pA}$ to $I_{\mathrm{h} 4 \mathrm{PPMA}}=24.9 \pm 8.0 \mathrm{pA}$ (at $-130 \mathrm{mV}, \mathrm{n}=7$, Wilcoxon signed-rank test $P=0.03$, Fig. 1B). Likewise, the time constant of $I_{\mathrm{h}}$ activation increased from $\tau_{\mathrm{ctrl}}=120.8 \pm$ $19.6 \mathrm{~ms}$ under control conditions to $\tau_{4 \beta \mathrm{PMA}}=153.1 \pm 23.2 \mathrm{~ms}$ during $1 \mu \mathrm{M} 4 \beta \mathrm{PMA}$ application ( $\mathrm{n}=7$, Wilcoxon signed-rank test $P=0.03$, Fig. 1D). Finally, as for rHCN1, 4ßPMA did not change the $\mathrm{V}_{1 / 2}$ of the hHCN1 mediated $I_{\mathrm{h}}\left(\mathrm{V}_{1 / 2 \mathrm{ctrl}}=-102.9 \pm 4.3 \mathrm{mV}\right.$ vs. $\mathrm{V}_{1 / 24 \beta \mathrm{PPA}}=-107.9 \pm$ $3.2 \mathrm{mV}, \mathrm{n}=7$, Wilcoxon signed rank test: $P=0.06$, Fig. $1 \mathrm{~F}$ ). This data suggest that reducing the amplitude of HCN1 mediated $I_{\mathrm{h}}$ without shifting the $V_{1 / 2}$ by $4 \beta \mathrm{PMA}$ in mammalian cells is independent of species-related differences and that PKC / PKC metabolites might not act via C-terminal loci that are non-homologous between both species (AA721 - AA748).

4ßPMA similarly affects rHCN1mediated I in neuroblastoma cells of the mouse (N1E-115)

The 4ßPMA effects on HCN1 presented so far might be HEK293-specific and not transferable to other mammalian cells including pyramidal neurons, in which HCN1 channels predominate. Therefore we studied the PKC effect on HCN1 in a more neuronal and assumingly less embryonic environment - the murine neuroblastoma cell line N1E-115.

By reproducing the cell attached measurements we showed that 4ßPMA similarly affected rHCN1 in N1E-115 cells and HEK293: 4ßPMA $(1 \mu \mathrm{M})$ reduced the maximal amplitude of rHCN1 mediated $I_{\mathrm{h}}$ to about $72 \%$ from $I_{\mathrm{hctrl}}=25.0 \pm 2.1 \mathrm{pA}$ to $I_{\mathrm{h} 4 \mathrm{BPMA}}=17.9 \pm 2.2 \mathrm{pA}(\mathrm{n}=7$, Wilcoxon-ranked test $P=0.02$, Fig. 2A/B) without shifting the $\mathrm{V}_{1 / 2}\left(\mathrm{~V}_{1 / 2 \mathrm{ctrl}}=-97.4 \pm 7.3 \mathrm{mV}\right.$ vs. $\mathrm{V}_{1 / 24 \text { 阝 PMA }}=-99.2 \pm 7.4 \mathrm{mV}, \mathrm{n}=7$, Wilcoxon signed rank test: $P=0.2$, Fig. $\left.2 \mathrm{~B}\right)$. The $I_{\mathrm{h}}$ activation slowed as indicated by the increase in activation time constant from $\tau_{\text {ctrl }}=73.4 \pm 26.7 \mathrm{~ms}$ under control conditions to $\tau_{4 \beta \mathrm{PMA}}=97.1 \pm 32.3 \mathrm{~ms}$ during $1 \mu \mathrm{M} 4 \beta \mathrm{PMA}$ application $(P=0.02$, Fig. 2B). The comparability of 4ßPMA effects on HCN1 in N1E-115 and HEK293 cells suggest that PKC act on HCN1 channels in mammalian cells independent of the cellular environment or developmental stage. 


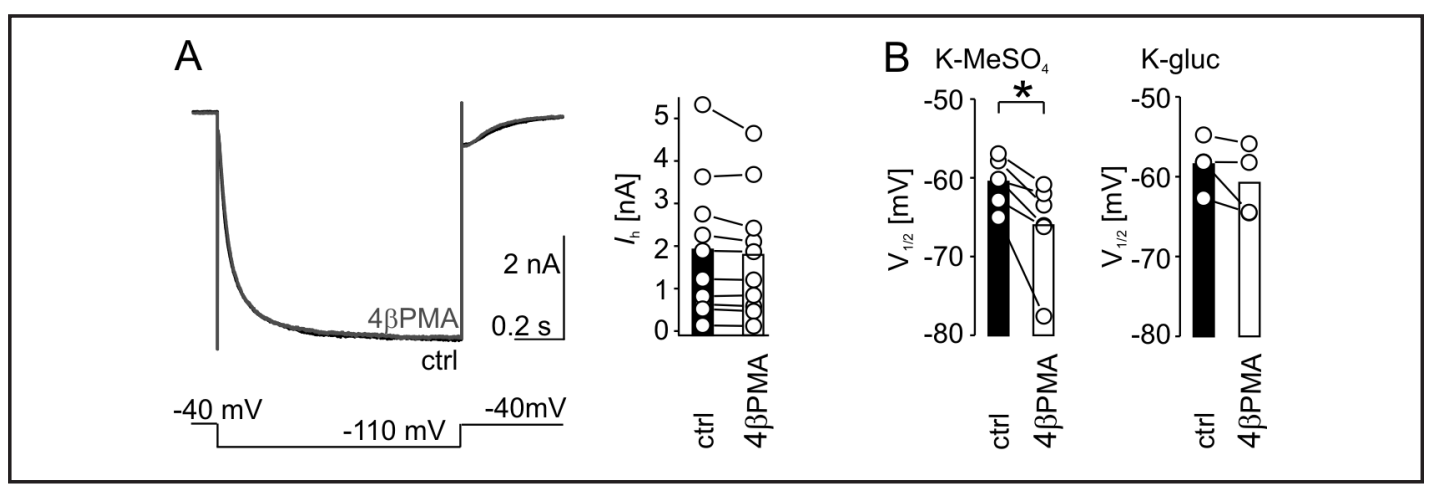

Fig. 3. Disruption of intracellular content impaired the 4ßPMA effects. A. Left: Traces of $I_{\mathrm{h}}$ in HEK293 cell expressing rHCN1 recorded in the whole cell mode at voltage steps to $-110 \mathrm{mV}$ with (grey traces) and without (black traces) $1 \mu \mathrm{M} 4 \beta$ PMA. Right: Population data of maximal $I_{\mathrm{h}}$ amplitude at $-110 \mathrm{mV}$. B. Voltage dependence of $I_{\mathrm{h}}$ activation estimated in whole cell configuration using K-MeSO $\mathrm{S}_{4}$ (left) or K-gluconate- (right) based intracellular solution. Individual Boltzmann fits of tail current fractions were used for estimating the plotted $\mathrm{V}_{1 / 2} \mathrm{~s}$.

An intact intracellular environment is a prerequisite for $4 \beta P M A$ to exert its modulatory effect on rHCN1 mediated $I_{h}$

To test whether 4 $\beta$ PMA modulation of rHCN1 channels requires an intact intracellular environment, we next applied $1 \mu \mathrm{M} 4 \beta \mathrm{PMA}$ during whole cell patch clamp recordings of rHCN1 transfected HEK293 cells. Such disruption of the proper intracellular environment prevented the main $4 \beta$ PMA effect: $4 \beta$ PMA did not change the maximum amplitude of $I_{\mathrm{h}}\left(I_{\text {hctrl }}\right.$ $=1.9 \pm 0.5 \mathrm{nA}$ to $I_{\mathrm{h} 4 \mathrm{\beta PMA}}=1.79 \pm 0.46 \mathrm{nA}, \mathrm{n}=10, P=0.11$; Fig. $3 \mathrm{~A}$ ). This prevention persisted when looking separately to experiments with differing intracellular solutions (see Materials and Methods, for K-gluconate based solution: $I_{\text {hctrl }}=1.9 \pm 0.8 \mathrm{nA}$ to $I_{\mathrm{h} 4 \mathrm{\beta PMA}}=1.85 \pm 0.8 \mathrm{nA}$, $\mathrm{n}=4, P=0.6 ; \mathrm{K}^{-\mathrm{MeSO}_{4}}$ based solution: $I_{\text {hctrl }}=1.9 \pm 0.7 \mathrm{nA}$ to $I_{\mathrm{h} 4 \beta \mathrm{PMA}}=1.75 \pm 0.6 \mathrm{nA}, \mathrm{n}=6, P$ $=0.09$ ). Further, 4ßPMA did not affect the $I_{\mathrm{h}}$ activation kinetics. As in our previous study [6], whole-cell recorded rHCN1 current activation better matched to a double exponential fit. Both time constants remained comparable under 4 $\beta$ PMA, when using K-gluconate based intracellular solution $\left(\tau_{1 \mathrm{ctrl}}=21.4 \pm 2.1 \mathrm{~ms}\right.$ vs. $\tau_{14 \beta \mathrm{PMA}}=25.9 \pm 4.1 \mathrm{~ms}, \mathrm{n}=4$, Wilcoxon signedrank test: $P=0.4 ; \tau_{2 \mathrm{ctrl}}=96.9 \pm 14.1 \mathrm{~ms}$ vs. $\tau_{24 \mathrm{BPMA}}=100.4 \pm 20.4 \mathrm{~ms}, \mathrm{n}=4, P=1$ ) at full current activation $(-110 \mathrm{mV})$. Also the voltage dependence of activation was stable under 4 $\beta$ PMA, when using K-gluconate based intracellular solution $\left(\mathrm{V}_{1 / 2 \mathrm{ctrl}}=-58.4 \pm 1.6 \mathrm{mV}\right.$ and $\mathrm{V}_{1 / 24 \beta \mathrm{PMA}}=$ $-60.8 \pm 2.2 \mathrm{mV}, \mathrm{n}=4, P=0.125$; Fig. 3B). However, during the $10 \mathrm{~min}$ of $4 \beta \mathrm{PMA}$ application the current activation slowed when recorded with $\mathrm{K}_{-} \mathrm{MeSO}_{4}$ based intracellular solution: $\tau_{1 \mathrm{ctrl}}$ $=19.21 \pm 4.7 \mathrm{~ms}$ vs. $\tau_{14 \mathrm{PPMA}}=27.4 \pm 2.5 \mathrm{~ms}, \mathrm{n}=6$, Wilcoxon signed-rank test, $P=0.06 ; \tau_{2 \text { ctrl }}$ $=78.76 \pm 13.3 \mathrm{~ms}$ vs. $\left.\tau_{24 \beta \mathrm{PMA}}=103.4 \pm 14.3 \mathrm{~ms}, \mathrm{n}=6, P=0.09\right)$. This might be explained by the slight but consistent shift to more hyperpolarized potentials. Here, the $V_{1 / 2}$ decreased from $\mathrm{V}_{1 / 2 \mathrm{ctrl}}=-60.5 \pm 1.23 \mathrm{mV}$ to $\mathrm{V}_{1 / 24 \mathrm{PPMA}}=-66.0 \pm 2.5 \mathrm{mV}, \mathrm{n}=6, P<0.05$; Fig. 3B). Because the latter changes in voltage sensitivity were restricted to recordings with $\mathrm{K}-\mathrm{MeSO}_{4}$ based intracellular solution and resemble the known "run down" of HCN-channels we attribute them to the recording conditions rather than to a $4 \beta \mathrm{PMA}$ effect. Such $\mathrm{K}-\mathrm{MeSO}_{4}$ induced $I_{\mathrm{h}}$ reduction could explain the gradual enhancement of the input resistance found in rat CA1 pyramidal neurons $[10,11]$. Taken together, the disruption of the intracellular environment prevented the 4ßPMA effects observed in intact cells.

$4 \beta P M A$ induced $I_{h}$ changes are mediated via PKC

We here used the high affinity stereoselective agonist of the cysteine-rich C-1

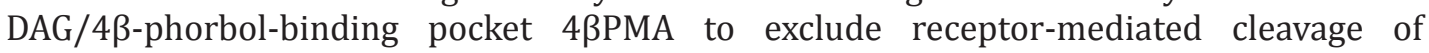
phosphatidylinositol 4,5-bisphosphate (4,5-PIP2) and changes in calcium signaling [4]. 


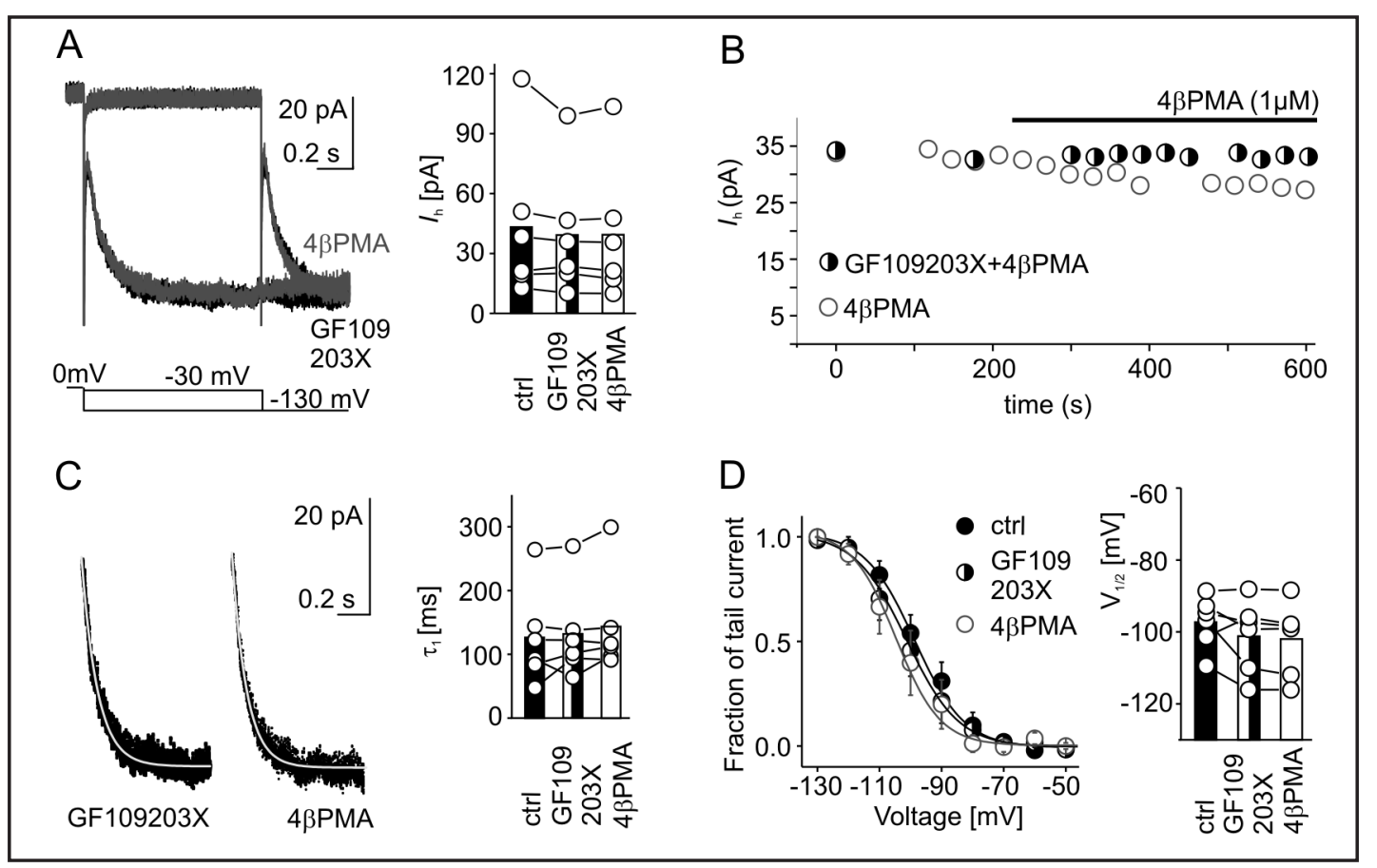

Fig. 4.The PKC inhibitor GF109203X prevented the 4ßPMA effects. A. Inhibiting PKC prevented the 4ßPMAinduced reduction of rHCN1 current amplitude when recorded in cell-attached mode. Left: rHCN1 mediated $I_{\mathrm{h}}$ at voltage steps to $-30 \mathrm{mV}$ and $-130 \mathrm{mV}$ in a HEK293 cell was comparable when $1 \mu \mathrm{M} 4 \beta \mathrm{PMA}$ (grey trace) was applied under PKC blockade by GF109203X (black trace). Right: Population data of maximal $I_{\mathrm{h}}$ amplitudes estimated as in Fig. 1A. B. Time course of $I_{\mathrm{h}}$ amplitudes at $-100 \mathrm{mV}$ following the application of $1 \mu \mathrm{M} 4 \beta \mathrm{PMA}$ (horizontal grey line) to untreated (open circles) or PKC blocked (GF109203X, halved black and white circles) in both cases exemplarily in one cell. C. Prevention of 4ßPMA-induced rHCN1 current activation alteration by PKC inhibition. Left: Distinct data points represent the time course of $I_{\mathrm{h}}$ activation at -130 mV during the application of $1 \mu \mathrm{M}$ GF109203X (left trace) and after additional $1 \mu \mathrm{M} 4 \beta \mathrm{PMA}$ application (right trace) with the respective single exponential function (light grey line). Right: Population data of time constants of $I_{\mathrm{h}}$ activation for each condition. D. Left: Voltage dependence of rHCN1 mediated $I_{\mathrm{h}}$ activation estimated and averaged over all patches as described in Fig. 1B. Right: Population data of individual $V_{1 / 2}$ estimated separately for each patch. Note, that also PKC inhibition itself (halved black and white circles and column) left all $I_{\mathrm{h}}$ parameters unchanged.

However, 4ßPMA may have a number of effects beyond PKC activation: it might also activate ERK, all three MAPK subfamilies, in particular p38 [12], Akt/PKB [13] or Rac2 [14], the latter two in a PI3K-independent manner. Therefore we tested whether 4 $\beta$ PMA modulated rHCN1-mediated $I_{\mathrm{h}}$ specifically via its PKC activation properties by sequentially applying the bisindolylmaleimide derivative GF109203X $(1 \mu \mathrm{M})$, a potent and selective inhibitor of PKC for $>10$ minutes followed by $4 \beta P M A$. Such treatment prevented the $I_{\mathrm{h}}$ amplitude reduction $\left(I_{\text {hGF } 109203 \mathrm{X}}=39.2 \pm 13.0 \mathrm{pA}\right.$ vs. $I_{\mathrm{h} 4 \beta \mathrm{PMA}}=39.2 \pm 14.0 \mathrm{pA}, \mathrm{n}=6$, Wilcoxon signed-rank test, $P=0.84$; Fig. 4A/B) in cell-attached recordings of HEK293 cells. It also impeded the 4ßPMA effect on the kinetics of $I_{\mathrm{h}}$ activation. Fitting $I_{\mathrm{h}}$ activation at the voltage step of $-130 \mathrm{mV}$ using a single exponential equation revealed no $4 \beta \mathrm{PMA}$ induced changes in the kinetics of the channel $\left(\tau_{\text {GF109203X }}=131.56 \pm 29.5 \mathrm{~ms}\right.$ vs. $\tau_{4 \beta \mathrm{PMA}}=143.12 \pm 32.1 \mathrm{~ms}, P=0.22$, Fig. $\left.4 \mathrm{C}\right)$. As expected from the initial cell attached recordings (Fig. $1 \mathrm{C}$ ), $4 \beta$ PMA did not alter the $V_{1 / 2}\left(V_{1 / 2 \mathrm{GF} 109203 \mathrm{X}}\right.$ $=-101.1 \pm 4.15 \mathrm{mV}$ vs. $\left.\mathrm{V}_{1 / 24 \beta \mathrm{PMA}}=-101.9 \pm 4.2 \mathrm{mV} P=0.09\right)$ when applied subsequent to GF109203X (Fig. 4D). However, the inhibition of basal PKC activity by GF109203X did not affect the maximal $I_{\mathrm{h}}\left(I_{\text {hctrl }}=43.4 \pm 15.8\right.$ pA vs. $I_{\text {hGF109203x }}=39.2 \pm 13.0 \mathrm{pA}, P=0.16$; Fig. $\left.4 \mathrm{~A}\right)$, voltage sensitivity $\left(\mathrm{V}_{1 / 2 \mathrm{ctrl}}=-97.3 \pm 2.98 \mathrm{mV}\right.$ vs. $V_{1 / 2 \mathrm{GF} 109203 \mathrm{X}}=-101.1 \pm 4.15 \mathrm{mV}, P=0.2$, Fig. 
4D) or time course of $I_{\mathrm{h}}$ activation $\left(\tau_{\mathrm{ctrl}}=125.86 \pm 30.8 \mathrm{~ms}\right.$ vs. $\tau_{\mathrm{GF} 109203 \mathrm{X}}=131.56 \pm 29.5 \mathrm{~ms}$, $P=0.8$; Fig. 4C), suggesting a rather low basal PKC activity in HEK293 under our conditions. Above results strengthen our argument that PKC activation modulates HCN1 mediated $I_{\mathrm{h}}$ and exclude a direct 4 $\beta$ PMA action of on HCN1 channels.

\section{Discussion}

Against the background of apparently opposing effects of PKC activation on HCN1 (and HCN2) mediated currents in Xenopus oocytes [4] we here investigated the effects of PKC

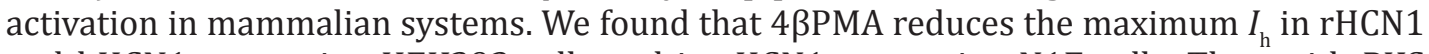
and hHCN1 expressing HEK293 cells and in rHCN1 expressing N1E cells. Therewith PKC activation overall inhibits $I_{\mathrm{h}}$ in mammalian cells in contrast to the at least partially activating effect in Xenopus oocytes [4]. As discussed in detail below, these changes are most likely attributable to the diverging expression systems, in particular to developmental (oocyte vs. more differentiated embryonic/neuroblastoma) stages or to phylogenetical (amphibian vs. mammal) origin.

The 4ßPMA effect in the mammalian cells was specifically attributable to PKC activation, since it was prevented by pre-incubation of the PKC inhibitor GF109203X and mediated by intracellular compounds. Basal PKC activity - despite putatively being influential in other systems - did not modulate the rHCN1 mediated $I_{\mathrm{h}}$ in HEK293 cells. The reduction of the maximal $I_{\mathrm{h}}$ qualitatively resembles the one found in Xenopus oocytes [4]. Although HCN1 channels may posses phosphorylation sites with a predicted high likelihood of interaction [5], a direct phosphorylation of the channel was rendered unlikely, because the 4ßPMA effects persisted when most cytoplasmatically exposed serines, threonines and tyrosines were removed by site directed mutagenesis [4]. Further following the convincing hypothesis of Fogle et al. [4], suppression of maximal $I_{\mathrm{h}}$ likely arises from membrane enrichment with proendocytotic acidic lipids such as arachidonic acid and / or phosphatidic acid followed by an enhanced retrieval of HCN channels from the plasma membrane [4]. Such dynamic regulation of surface expression also underlies the activity dependent HCN1 regulation $[15,16]$. Taken together, a reduction in the number of available channels seems plausible to explain the reduction of the maximal $I_{\mathrm{h}^{\prime}}$ however, our data cannot exclude changes in single channel current or open probability.

In contrast to the findings in Xenopus oocytes [4], the gating of neither rHCN1 nor hHCN1 channels was affected by 4ßPMA in HEK293 or N1E-115 cells. We can not exclude that this difference between the expression systems is in part due to the more depolarized basic value of the $\mathrm{V}_{1 / 2}$ in the Xenopus oocytes and therewith a more "active state" of the mHCN1 channels expressed in Xenopus oocytes ( $-66 \mathrm{mV}$ vs. -95 to $-100 \mathrm{mV}$ in this study). Reasons for variations in $V_{1 / 2}$ are manifold (partially discussed in Battefeld et al., [6]). However, following the above mentioned hypothesis reduction of maximal $I_{\mathrm{h}}$ [4] due to an increase in at least one of the acidic lipids would imply a shift of the channels voltage sensitivity. The lack of shift in the mammalian systems investigated by us might be due to HCN channel "context dependence" [17] suggested by different properties of identical HCN2/4 channels expressed in different systems [18]. Indeed, both expression systems (HEK293 and Xenopus oocytes) differ in many putatively relevant aspects: Whereas a large number of PKC isoforms are present in Xenopus oocytes (PKC $\alpha, \beta, \delta, \zeta, \varepsilon, \eta, \theta, \mu)[19,20]$, only some (PKC $\alpha, \beta, \delta, \varepsilon)$ were detected endogenously in HEK293 [21].

Distinctions in intracellular content might further result in different interaction with essential downstream players as diacylglycerol kinase or cytoplasmic/independent phospholipaseA2, finally leading to a lack of PKC induced increase in the levels of phosphatidic acid and arachidonic acid or an imbalance of both. Although tempting, it is impossible to pin down the effect to either of these major pathways, because both were necessary for the effects on gating and maximal current, as shown in the blocking experiments [4]. Therefore, it seems more likely that the "direct" interaction of acidic lipids with the channels differs 
between the expression systems, maybe due to varying conditions in the channel vicinity, e. g. the availability of putatively required additional proteins [as for p38MAPK - 22]. Finally, even though we ensured the rHCN1 sequence, we cannot exclude different HCN1 protein modulations by posttranslational modifications and/or protein-protein interactions [23, 24].

Although our results in HEK293 and N1E-115 cells are not directly transferrable, it is interesting to note that PKC activation inhibits $I_{\mathrm{h}}$ in several regions of the central nervous system. As in HEK293 cells PKC reduced the maximal conductance of mainly HCN2/3 containing channels when activated by neurotensin receptors in the substantia nigra [25], and of mainly HCN1 containing channels in pyramidal neurons following noradrenergic receptor stimulation in the prefrontal cortex [26] or following metabotrobic glutamate receptor stimulation in the hippocampus [11], notably without evidence for a change in the voltage dependence of activation. Such PKC-induced modulation of $I_{\mathrm{h}}$ in somatosensory neurons might - if present - link the IFN signaling to neuronal excitability changes [7]. Notably, biophysical characteristics of HCN1 channels expressed in HEK293 cells (in particular their $\mathrm{V}_{1 / 2}$ ) are closely related to neuronal HCN channels when recorded at dendrites of pyramidal cortical and hippocampal neurons in cell attached mode $[9,27,28]$. This apparent phenomenological resemblance might specify HEK293 or N1E-115 cells as model systems to further elucidate neuronal mechanisms of PKC actions.

\section{Acknowledgements}

We thank Arne Battefeld, Konstantin Stadler, Roland Bender and Olaf Ninnemann for valuable comments, Lennart Barthel for recording from N1E-115 cells and Julia König for technical assistance. The German Research Foundation DFG (STR865/3-1) and the Sonnenfeld Stiftung supported the study.

\section{References}

1 Wahl-Schott C, Biel M: HCH channels: Structure, cellular regulation and physiological function. Cell Mol Life Sci 2009;66:470-494.

2 Lorincz A, Notomi T, Tamas G, Shigemoto R, Nusser Z: Polarized and compartment-dependent distribution of HCN1 in pyramidal cell dendrites. Nat Neurosci 2002;5:1185-1193.

- Zolles G, Klocker N, Wenzel D, Weisser-Thomas J, Fleischmann BK, Roeper J, Fakler B: Pacemaking by HCN channels requires interaction with phosphoinositides. Neuron 2006;52:1027-1036.

4 Fogle KJ, Lyashchenko AK, Turbendian HK, Tibbs GR: HCN pacemaker channel activation is controlled by acidic lipids downstream of diacylglycerol kinase and phospholipase A2. J Neurosci 2007;27:2802-2814.

5 Poolos NP, Bullis JB, Roth MK: Modulation of h-channels in hippocampal pyramidal neurons by p38 mitogenactivated protein kinase. J Neurosci 2006;26:7995-8003.

6 Battefeld A, Bierwirth C, Li YC, Barthel L, Velmans T, Strauss U: I(h) "run-up" in rat neocortical neurons and transiently rat or human HCN1-expressing HEK293 cells. J Neurosci Res 2010;88:3067-3078.

-7 Stadler K, Bierwirth C, Stoenica L, Battefeld A, Reetz O, Mix E, Schuchmann S, Velmans T, Rosenberger K, Brauer AU, Lehnardt S, Nitsch R, Budt M, Wolff T, Kole MH, Strauss U: Elevation in type I interferons inhibits HCN1 and slows cortical neuronal oscillations. Cereb Cortex 2012; DOI: 10.1093/cercor/bhs305.

-8 Gill CH, Randall A, Bates SA, Hill K, Owen D, Larkman PM, Cairns W, Yusaf SP, Murdock PR, Strijbos PJLM, Powell AJ, Benham CD, Davies CH: Characterization of the human HCN1 channel and its inhibition by capsazepine. Br J Pharmacol 2004;143:411-421

-9 Kole MHP, Hallermann S, Stuart GJ: Single Ih channels in pyramidal neuron dendrites: Properties, distribution, and impact on action potential output. J Neurosci 2006;26:1677-1687.

10 Kaczorowski CC, Disterhoft J, Spruston N: Stability and plasticity of intrinsic membrane properties in hippocampal CA1 pyramidal neurons: Effects of internal anions. J Physiol 2007;578:799-818. 


\section{Cellular Physiology Cell Physiol Biochem 2013;31:532-541

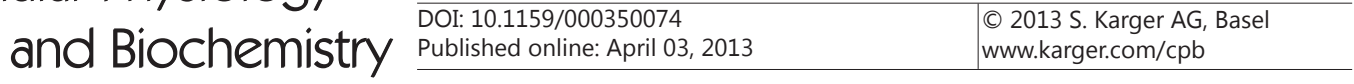 \\ Reetz/Strauss: PKC Mediated HCN1 Inhibition}

11 Brager DH, Johnston D: Plasticity of intrinsic excitability during long-term depression is mediated through mglur-dependent changes in I(h) in hippocampal CA1 pyramidal neurons. J Neurosci 2007;27:13926-13937.

-12 Simon C, Goepfert H, Boyd D: Inhibition of the p38 mitogen-activated protein kinase by SB 203580 blocks PMA-induced Mr 92,000 type IV collagenase secretion and in vitro invasion. Cancer Res 1998;58:1135-1139.

$\checkmark 13$ Kubohara Y, Hosaka K: The putative morphogen, DIF-1, of dictyostelium discoideum activates Akt/PKB in human leukemia K562 cells. Biochem Biophys Res Commun 1999;263:790-796.

14 Akasaki T, Koga H, Sumimoto H: Phosphoinositide 3-kinase-dependent and -independent activation of the small GTPase Rac2 in human neutrophils. J Biol Chem 1999;274:18055-18059.

15 Arimitsu T, Nuriya M, Ikeda K, Takahashi T, Yasui M: Activity-dependent regulation of HCN1 protein in cortical neurons. Biochem Biophys Res Commun 2009;387:87-91.

-16 Fan Y, Fricker D, Brager DH, Chen X, Lu HC, Chitwood RA, Johnston D: Activity-dependent decrease of excitability in rat hippocampal neurons through increases in I(h). Nat Neurosci 2005;8:1542-1551.

17 Baruscotti M, Bucchi A, DiFrancesco D: Physiology and pharmacology of the cardiac pacemaker ("funny") current. Pharmacol Ther 2005;107:59-79.

18 Qu JH, Altomare C, Bucchi A, DiFrancesco D, Robinson RB: Functional comparison of HCN isoforms expressed in ventricular and HEK293 cells. Pflugers Arch 2002;444:597-601.

19 Gundersen CB, Kohan SA, Chen Q, Iagnemma J, Umbach JA: Activation of protein kinase Ceta triggers cortical granule exocytosis in Xenopus oocytes. J Cell Sci 2002;115:1313-1320.

-20 Johnson J, Capco DG: Progesterone acts through protein kinase C to remodel the cytoplasm as the amphibian oocyte becomes the fertilization-competent egg. Mech Dev 1997;67:215-226.

-21 Kawasaki T, Ueyama T, Lange I, Feske S, Saito N: Protein kinase C-induced phosphorylation of Orai1 regulates the intracellular $\mathrm{Ca}^{2+}$ level via the store-operated $\mathrm{Ca}^{2+}$ channel. J Biol Chem 2010;285:25720-25730.

-22 Levitan IB: Signaling protein complexes associated with neuronal ion channels. Nat Neurosci 2006;9:305310.

-23 Santoro B, Wainger BJ, Siegelbaum SA: Regulation of HCN channel surface expression by a novel c-terminal protein-protein interaction. J Neurosci 2004;24:10750-10762.

24 Huang J, Huang A, Zhang Q, Lin YC, Yu HG: Novel mechanism for suppression of hyperpolarization-activated cyclic nucleotide-gated pacemaker channels by receptor-like tyrosine phosphatase-alpha. J Biol Chem 2008;283:29912-29919.

25 Cathala L, Paupardin-Tritsch D: Neurotensin inhibition of the hyperpolarization-activated cation current (Ih) in the rat substantia nigra pars compacta implicates the protein kinase C pathway. J Physiol 1997;503:87-97.

26 Carr DB, Andrews GD, Glen WB, Lavin A: Alpha2-noradrenergic receptors activation enhances excitability and synaptic integration in rat prefrontal cortex pyramidal neurons via inhibition of HCN currents. J Physiol 2007;584:437-450.

-27 Atkinson SE, Williams SR: Postnatal development of dendritic synaptic integration in rat neocortical pyramidal neurons. J Neurophysiol 2009;102:735-751.

28 Dougherty KA, Nicholson DA, Diaz L, Buss EW, Neuman KM, Chetkovich DM, Johnston D: Differential expression of HCN subunits alters voltage-dependent gating of h-channels in CA1 pyramidal neurons from the dorsal and ventral hippocampus. J Neurophysiol 2013; DOI:10.1152/jn.00010.2013. 